Pacific Journal of Mathematic 


\title{
ARITHMETIC PROPERTIES OF THE IDÈLE DISCRIMINANT
}

\author{
DONALD MAURER
}

A theorem of Hecke asserts that the discriminant $D_{K / F}$ of an extension of algebraic number fields $K / F$ is a square in in the absolute class group. In 1932 Herbrand conjectured the following related theorem and was able to prove it for metacyclic extensions: If $K / F$ is normal, then $\mathfrak{D}_{K / F}$ can be written in the form $\mathfrak{A}^{2}(\theta), \theta \in F$; where (i) $\theta \equiv 1$ $(\bmod \mathfrak{B}), \mathfrak{B}$ is the greatest divisor of 4 which is prime to $\grave{D}_{K / F}$, and (ii) $\theta>0$ at each real prime $v$ except when $K \otimes_{F} F_{v}$ is a direct sum of copies of the complex field and $(K: F) \equiv$ $2(\bmod 4)$.

More recently, A. Fröhlich gave a unified treatment of these and related questions using the concept of an idèle discriminant. The purpose of this paper is to present a generalization of these results with some connections with the structure of the Galois group.

Our notation will be as follows. Let $\mathscr{M}_{F}$ denote the finite prime divisors of $F$. The ring of integers in $F$ will be denoted by $\mathfrak{O}$ (or $\mathfrak{O}_{F}$ ), and for each $v \in \mathscr{M}_{F}$, $\mathcal{O}_{0}$ will be the integers of the completion $F_{v}$. Also, for $\alpha \in F_{v}$ we write $v(\alpha)$ for the order of $\alpha$, so that if the prime ideal $\mathfrak{\beta}_{\nu}$ of $\mathfrak{D}_{v}$ is generated by $\pi_{v}$, then $v\left(\pi_{v}\right)=1$. If $x$ is an idèle with $v$-component $x_{v}$, then we shall write $x=\left(x_{v}\right)$, and $v(x)=$ $v\left(x_{v}\right)$. If $\alpha \in F^{*}$ then, unless otherwise stated, $(\alpha)$ will denote the principal idèle defined by $\alpha_{v}=\alpha$. The idèle group $J_{F}$ contains, as a subgroup, the unit idèles $U_{F}$ consisting of those $x$ such that $x_{v} \in U_{v}$, the unit group in $F_{v}$, for all $U$. The idèle discriminant $d(K / F)$ defined in [1] is an element of $J_{F} / U_{F}^{2}$. The classical ideal discriminant is simply the ideal naturally determined by $d(K / F)$.

1. The general theory. Throughout the paper, $p$ will be a fixed prime, and we shall assume that $F$ contains $\zeta_{p}$, a primitive $p$ th-root of unity.

Our first results pertain to the case of cyclic $p$-extensions $K / F$. Let $G$ denote the Galois group.

LEMMA 1.1. Let $K / F$ be cyclic of degree $p$. Then there is an element $\alpha \in F$ such that $K=F\left(\alpha^{1 / p}\right)$ and $\alpha \equiv 1(\bmod \mathfrak{B})$, where $\mathfrak{B}$ is the greatest divisor of $\left(\zeta_{p}-1\right)^{p}$ which is relatively prime to the discriminant $\delta_{K / F}$. Moreover, $v$ splits in $K$ if and only if $\alpha \in F^{p}$. 
Proof. For each $v \in \mathscr{A}_{F}$, let $K_{\nu}=K \bigotimes_{F} F_{v}$; then $K_{v}$ is algebraisomorphic to a direct product $\Pi_{\omega / v} K_{\omega}$ of local field extensions $K_{\omega} / F_{\nu}$. Similarly, if we let $\left(\mathfrak{V}_{K}\right)_{v}=\mathfrak{D}_{K} \boldsymbol{\bigotimes}_{0} \mathfrak{D}_{\nu}$, then $\left(\mathfrak{D}_{K}\right)_{v}=\Pi_{\omega / \nu} \mathfrak{D}_{\omega}$. Let $\mathscr{P}$ be the set of all $v$ which divide $p$ (i.e., $v(p)>0$ ) but do not divide $\mathfrak{d}_{K / F}$. Then for each $v \in \mathscr{P}, K_{v}$ is nonramified, and so $K_{v}$ has a normal $\mathfrak{D}_{\nu}$-integral basis $\left\{x_{g}^{(v)}\right\}_{g \in G}$. By the strong approximation theorem, it is then possible to find a normal $F$-basis $\left\{x_{g}\right\}_{g \in G}$ of $K$ which is an $\mathfrak{D}_{v}$-integral basis of $\left(\mathfrak{D}_{K}\right)_{v}$ at each $v \in \mathscr{P}$. Moreover, we may also assume that $\sum_{g \in G} x_{g}=1$.

Now for each character $\chi_{:} G \rightarrow C$ (the complex field) set $\theta_{\chi}=$ $\sum_{g \in G} \chi(g) x_{g}$. It is well known that $\alpha_{\chi}=\theta_{\chi}^{p} \in F$, and $K=F\left(\alpha^{1 p /}\right)$ for a nontrivial $\chi$. Fix such a $\chi$, and write $\alpha=\alpha_{\chi}$. We have

$$
\theta_{\chi}=1+\sum_{g \neq 1}(\chi(g)-1) x_{g} .
$$

But in the field $\boldsymbol{Q}_{p}$ of the $p$ th roots of unity over the rational field we can write

$$
\chi(g)-1=c_{\chi}\left(\zeta_{p}-1\right) \quad\left(c_{\chi} \text { integral in } \boldsymbol{Q}_{p}\right) .
$$

Hence $\theta_{\chi}=1+h_{\chi}^{\prime}\left(\zeta_{p}-1\right)$ with $h_{\alpha}^{\prime} \in K$. It follows that $\alpha=1+$ $h_{x}\left(\zeta_{p}-1\right)^{p}$ with $h_{x} \in F$. Moreover, if $v \in \mathscr{P}$, then $h_{x} \in \mathfrak{O}_{v}$. Thus the lemma is proved.

We continue to suppose that $K / F$ is a cyclic $p$-extension. For $v \in \mathscr{l}_{F}$, let $G_{i}$ denote the $i$ th ramification group of a localization $K_{\omega} / F_{v}$. We define the ramification number $r_{\nu}$ to be the smallest integer $n$ such that $G_{n}$ is trivial. Clearly $r_{v}$ is independent of $\omega$. Now $v$ is nonramified, tamely ramified, or wildly ramified according as $r_{v}=0, r_{v}=1$ or $r_{v}>1$ respectively. If $(K: F)=p$, then the ramification numbers $r$, give a complete description of ramification, and $v(d(K / F))=r_{v}(p-1)$.

The next lemma gives a partial determination of the ramification numbers $r$, when $(K: F)=p$.

Lemma 1.2. Suppose $K=F\left(\alpha^{1 / p}\right)$ with $\alpha \in F$. If $v$ is ramified and $v(\alpha) \not \equiv 0(\bmod p)$, then $r_{0}=1$ or $v\left(\zeta_{p}-1\right) p+1$.

Proof. Set $s=v\left(\zeta_{p}-1\right)$. If $v$ is tamely ramified, the lemma is obvious. Therefore we may suppose that $v$ is wildly ramified; so $p$ divides the ramification number of $v$ when extended to $K$, but $v(\alpha) \not \equiv 0(\bmod p)$. Then let $\alpha^{1 / p}=\varepsilon \pi_{\omega}^{a}$, where $\omega$ is the extension of $v$ to $K, \pi_{\omega}$ a local prime, $\varepsilon \in U_{\omega}$ and $(a, p)=1$. Now there is a $\gamma \in U_{\nu}$ such that $\zeta_{p}=1-\gamma \pi_{\nu}^{s}$, and an element $\sigma \in \operatorname{Gal}\left(K_{\omega} / F_{v}\right)$ such that 


$$
\zeta_{p}=\frac{\sigma\left(\alpha^{1 / p}\right)}{\alpha^{1 / p}}=\left(\frac{\sigma\left(\pi_{\omega}\right)}{\pi_{\omega}}\right)^{a} \frac{\sigma(\varepsilon)}{\varepsilon} .
$$

Since $\pi_{\omega}^{r}\left(r=r_{v}\right)$ is the highest power of $\pi_{\omega}$ which divides $\sigma\left(\pi_{\omega}\right)-\pi_{\omega}$, it follows that

$$
\frac{\sigma\left(\pi_{\omega}\right)}{\pi_{\omega}} \in U_{r-1}-U_{r}
$$

where $U_{m}=1+\Re_{v}^{m}$. Since $(a, p)=1$, it is also true that

$$
\left(\frac{\sigma\left(\pi_{\omega}\right)}{\pi_{\omega}}\right)^{a} \in U_{r-1}-U_{r} .
$$

But $\sigma(\varepsilon) / \varepsilon \in U_{r}$, whence it follows that $\zeta_{p}=1-\gamma^{\prime} \pi_{\omega}^{p s}$ belongs to $U_{r-1}-U_{r}$.

This completes the proof.

It is not possible to say much about $r_{\diamond}$ when $v(\alpha) \equiv 0(\bmod p)$. A slight modification of the previous argument shows that $r_{v} \leqq s p$. However, if $n$ is any integer in the range $0<n \leqq s p$, then according to [5] or [7], for a $y \in F_{0}$ with $v(y)=1-n$, the roots of

$$
x^{p}-x-y=0
$$

generate a cyclic extension of derree $p$ with $r_{v}=n$.

Let $K / F$ be cyclic of degree $p$, then a divisior $v \in \mathscr{M}_{F}$ will be called exceptional at $K / F$ if the congruence $v(\alpha) \cdot x \equiv r,(\bmod p)$ does not have a solution relatively prime to $p$. That is, $v$ is exceptional if one, but not both, of $v(\alpha)$ or $r$ is congruent to $0(\bmod p)$. Suppose $v(\alpha) \not \equiv 0(\bmod p)$, but $r_{s} \equiv 0(\bmod p)$. By Lemma $1.2 r_{v}=0$, and so $K_{v} / F_{v}$ is nonramified. Since $\alpha$ is a $p$ th power in $K, p$ must divide $v(\alpha)$, a contradiction. Hence $v$ is exceptional if and only if it is totally ramified, and $v(\alpha) \cdot x \equiv r(\bmod p)$ is not solvable, i.e., $v(\alpha) \equiv$ $0(\bmod p)$ but $r, \not \equiv 0(\bmod p)$.

Now let $K / F$ be any finite Galois extension such that $(K: F)$ is divisible by $p$. In order to state the main theorem, it will be convenient to introduce two functions $\phi_{K / F}$ and $\psi_{K / F}$ on $\mathscr{A}_{F}$. Suppose $K / F$ is a $p$-extension, and let $T$ be a subfield such that $(K: T)=p$. We define $\phi_{K / F}(U)=0$ unless $v$ is totally ramified in $K / F$, and $K / T$ is exceptional at the extension $\omega$ of $v$ to $T$. In the latter case, $\phi_{K / F}(v)$ is to be the least positive residue $(\bmod p)$ of $-r_{\omega}$. This definition is independent of the choice of $T$. For suppose that $T^{\prime}$ also satisfies the condition $\left(K: T^{\prime}\right)=p$. We may suppose that $v$ is totally ramified. The tower formula applied to the localization at $v$ gives (since $v$ is totally ramified, we can identify $\omega$ and $v$ when convenient)

$$
N\left(d\left(K_{v} / T_{v}\right)\right) \equiv N^{\prime}\left(d\left(K_{v} / T_{\nu}^{\prime}\right)\right)(\bmod p),
$$


where $N$ and $N^{\prime}$ are the obvious norm maps. Recalling that $\omega(d(K / T))=r_{\omega}(p-1)$, this congruence then implies $\phi_{K / F}$ is well defined.

Now we extend our definition to the general case by letting $L$ be the fixed field of a $p$-Sylow group $G_{p}$. We define $\phi_{K / F}$ to be the least nonnegative residue $(\bmod p)$ of the expression $(L: F) \phi_{K / L}(\omega) / e_{L / F}(v)$, where $\omega$ extends $v$ to $L$, and $e_{L / F}(v)$ denotes the ramification index of $v$ in $L$. Again, it can be verified that this definition is independent of the choice of either $L$ or $\omega$. If $K / F$ is finite Galois, we say $v$ is exceptional at $K / F$ if $\phi_{K / F}(v) \neq 0$. This extends the earlier definition.

The function $\psi$ is defined in a similar manner. If $(K: F)=p$, then $\psi_{K / F}(v)=1$ for all exceptional $v$. Otherwise $\psi_{K / F}(v)$ is the least positive residue $(\bmod p)$, satisfying the congruence $v(\alpha) \cdot \psi_{K / F}(v) \equiv$ $r_{v}(\bmod p)$, where $K=F\left(\alpha^{1 / p}\right)$. In the general case, if $G_{p}$ is cyclic, let $T$ be a subfield such that $(K: T)=p$ and define $\psi_{K / F}(\nu)=\psi_{K / T}(\omega)$, where $\omega$ extends $v$ to $T$. If $G_{p}$ is noncyclic, define $\psi_{K / F}(v)=1$ for all $v$. The definition is independent of $T, \omega$ or $\alpha$. We can now state the main theorem of this section.

THEOREM 1.3. ${ }^{1}$ Let $\zeta_{p} \in F$, and suppose $K / F$ is a finite Galois extension whose group $G$ contains a nontrivial $p$-Sylow group $G_{p}$. Then there are ideles $a, b$ and $c$ in $J_{F}$ such that

$$
d(K / F) \equiv a^{p} b c\left(\bmod U_{F}^{2}\right) .
$$

Moreover, the following conditions are satisfied for all $v \in \mathscr{M}_{F}$.

(i) $c_{v}=\theta^{\psi(\nu)}\left(\psi=\psi_{K / F}\right)$ for some $\theta \in F$ satisfying the congruence $\theta \equiv 1(\bmod \mathfrak{B})$, where $\mathfrak{B}$ is the greatest divisor of $\left(\zeta_{p}-1\right)^{p}$ which is prime to $\delta_{K / F}$.

(ii) If $\cup$ is exceptional, $v(c) \equiv 0(\bmod p)$

(iii) If $G_{p}$ is noncyclic, then $\theta=1$. Moreover, if $K / F$ is a cyclic p-extension, a nonramfied $v$ prime to $p$ splits in $K / F$ if and only if $\theta \in U_{u}^{p}$.

(iv) $b_{\nu}=\pi_{0}^{\phi(v)}\left(\phi=\phi_{K / F}\right)$.

We do not deal with the infinite components of $d(K / F)$, for when $p=2$ this is discussed in [1]; and for $p>2, F_{v}=C$ for all infinite $v$, whence $d(K / F)$, is trivial. The remainder of this section is devoted to proving the theorem, while in the final section some consequences are discussed. In particular, the case $p=2$ is developed.

We first deal with $p$-extensions, so let $(K: F)=p^{m}$. If $m=1$, let $K=F\left(\alpha^{1 / p}\right)$, where $\alpha$ satisfies the congruence condition of Lemma

${ }^{1}$ Results of a similar nature, although somewhat weaker, can be proved without the restriction $\zeta_{p} \in F$. 
1.1. A field basis for $K$ is then $1, \gamma, \gamma^{2}, \cdots, \gamma^{p-1}$ with $\gamma=\alpha^{1 / p}$. Therefore $d(K / F)$ will have a local representation at $v$ of the form

$$
d(K / F)_{v} \equiv(-1)^{p(p-1) / 2} p^{p} \beta_{l}^{2} \alpha^{p-1}\left(\bmod U_{v}^{2}\right),
$$

for some $\beta_{u} \in F_{u}$. Using the relation $v(d(K / F))=r_{u}(p-1)$, this gives the congruence $2 v\left(\beta_{v}\right) \equiv-r_{\iota}+v(\alpha)(\bmod p)$. Hence there is a function $\dot{\phi}_{K / F}^{\prime}$ which satisfies the congruence equation $2 \phi^{\prime} \equiv \phi(\bmod p)$. In particular if $p=2$, then $\phi \equiv 0$ and so there are no exceptional primes. Now if $v$ is exceptional, then our result implies that $\beta_{v}=\varepsilon, \pi_{v}^{\phi(v)}$ for some unit $\varepsilon_{v}$. On the other hand, if $v$ is nonexceptional, then $v(\alpha) \cdot \psi(\nu) \equiv r_{\nu}(\bmod p)$. Therefore in the above representation for $d(K / F)_{0}$ we can replace $\alpha$ by $\alpha^{\psi(v)}$. Again, $\beta_{v}$ is of the form $\varepsilon_{\imath} \pi_{v}^{\phi^{\prime}(v)}$. Thus we obtain the global idèle representation

$$
d(K / F) \equiv \delta^{p} \beta^{2} \tau^{p-1}\left(\bmod U_{F}^{2}\right),
$$

where each component of $\beta$ is given by $\beta_{v}=\varepsilon, \pi_{0}^{\phi^{\prime}(v)}$, and $\tau_{v}=\alpha^{\psi(v)}$. Moreover, for all nonramified $v, \alpha \in U_{v}^{p}$ if and only if $v$ splits in $K$.

This representation can be generalized to any cyclic $p$-extension. There is a sequence of subfields

$$
F=\Omega_{0} \subset \Omega_{1} \cdots \subset \Omega_{r} \subset \Omega_{r+1}=K
$$

with $\left(\Omega_{i}: \Omega_{i-1}\right)=p$. For notational simplicity we set $T=\Omega_{r}$. According to our previous arguments, we have the representation $d(K / T) \equiv \delta_{T}^{p} \beta_{T}^{2} \tau_{T}^{p-1}\left(\bmod U_{T}^{2}\right)$. The tower formula gives $d(K / F) \equiv$ $\delta^{p} \beta^{2} \tau^{p-1}\left(\bmod U_{F}^{2}\right)$, where $\beta=N_{T / F}\left(\beta_{T}\right)$ and $\tau=N_{T / F}\left(\tau_{T}\right)$. By a straightforward computation, $\beta_{v}=\varepsilon_{v} \pi_{v}^{\phi^{\prime}(v)}\left(\phi^{\prime}=\phi_{K / F}^{\prime}\right)$. Similarly, if we define $\alpha=N_{T / F}\left(\alpha_{T}\right)$, then $\tau_{v}=\alpha^{\psi(0)}\left(\psi=\psi_{K / F}\right)$.

Suppose that $v$ divides $p$ but not $\delta_{K / F}$. Then an extension $\omega$ of v to $T$ also divides $p$ but not $\mathfrak{D}_{K / T}$; therefore in $\mathfrak{O}_{\omega}, \alpha_{T}=1+$ $h_{\omega}\left(\zeta_{p}-1\right)^{p}$. Since $T / F$ is normal, we have

$$
N_{\omega / \nu}\left(\alpha_{T}\right)=\prod_{\sigma}\left(1+\sigma\left(h_{\omega}\right)\left(\zeta_{p}-1\right)^{p}\right),
$$

where $\sigma$ runs through the elements of the Galois group of $T_{\omega} / F_{v}$. Hence it follows that $\alpha=1+h_{\nu}\left(\zeta_{p}-1\right)^{p}$ is in $\mathfrak{O}_{\nu}$.

Now we must show that if $v$ is nonramified in $K$, and prime to $p$, then $v$ splits if and only if $\alpha \in U_{v}^{p}$. Suppose that such a $v$ does not split in $K$. Then $\alpha_{T} \notin T_{0}^{p}$. In general if $U_{i}$ denotes the unit group of $\left(\Omega_{i}\right)_{*}$, we have $\left(U_{i}: U_{i}^{p}\right)=p$, so that the norm map induces an isomorphism $U_{i+1} / U_{i+1}^{p} \cong U_{i} / U_{i}^{p}$; hence $\alpha \notin U_{v}^{p}$. Since there are infinitely many primes which do not split in $K / F, \alpha$ cannot be a $p$ th power, and therefore $\left(F\left(\alpha^{1 / p}\right): F\right)=p$.

Now a nonramified $v$ will split in $K / F$ if and only if it splits 
in $\Omega_{1} / F$, for if it splits in $K$, then the decomposition field contains $\Omega_{1}$, whence $v$ also splits in $\Omega_{1} / F$. Hence if $\nu$ splits in $\Omega_{1}$, then it splits in $K$ and so also in $F\left(\alpha^{1 / p}\right)$; therefore $F\left(\alpha^{1 / p}\right)=\Omega_{1}$. This proves our assertion, and extends the representation of the idèle discriminant to arbitrary cyclic $p$-extensions.

Suppose now that $K / F$ is a noncyclic $p$-extension. The Galois group $G$ must contain a proper noncyclic subgroup. For suppose a maximal subgroup $N$ is cyclic. Let $a$ be a generator of $N$ and choose $b$ not in $N$. Then $p$ is the smallest positive integer $m$ such that $b^{m} \in N$. It follows that $G$ is generated by $a$ and $b$. The subgroup generated by $a^{p}$ and $b$ is proper and noncyclic. By a simple induction argument we conclude that $G$ contains a subgroup $H$ of type $(p, p)$.

Let $L$ be the fixed field of $H$. Then there is a subfield $K \supset$ $T \supset L$ such that $K=T\left(\mu^{1 / p}\right)$ with $\mu \in L$. As before, $d(K / T)$ has a representation of the form $\delta_{T}^{p} \beta_{T}^{2} \tau_{T}^{p-1}$, where each component of $\tau_{T}$ is a power of $\mu$. Since $N_{T / L}(\mu)=\mu^{p}$, the tower formula gives for each $\omega \in \mathscr{M}_{L}$ the representation $d(K / L)_{\omega} \equiv \delta_{\omega}^{p} \beta_{\omega}^{2}\left(\bmod U_{\omega}^{2}\right)$, where $\beta_{\omega}=$ $\varepsilon_{\omega} \pi_{\omega}^{\phi^{\prime}(\omega)}\left(\phi^{\prime}=\phi_{K / L}^{\prime}\right)$. The tower formula applied to $K \supset L \supset F$ then gives a representation of $d(K / F)$ of the form $\delta^{p} \beta^{2} \tau^{p-1}$, with $\beta_{0}=$ $\varepsilon_{v} \pi_{0}^{\phi(v)}$ and $\tau_{v}=1$ for all $v \in \mathscr{K}_{F}$.

This representation generalizes to arbitrary extensions $K / F$ by applying the tower formula to $K \supset L \supset F$, where $L$ is the fixed field of a $p$-Sylow subgroup of $G$. To obtain the theorem, we now take $b_{v}=\pi_{v}^{\phi(j)}$ and $\theta=\left(N_{L / F}(\alpha)\right)^{p-1}$, or $\theta=1$ depending on whether $G_{p}$ is cyclic or noncyclic. If $G_{p}$ is cyclic, then

$$
\begin{aligned}
\nu(c) & \equiv-\psi(\nu) \cdot v\left(N_{L / F}(\alpha)\right) \\
& \equiv \frac{(L: F)}{e_{L / F}(v)}(-\psi(\omega) \cdot \omega(\alpha))(\bmod p),
\end{aligned}
$$

where $\omega$ extends $v$ to $L$. For an exceptional $), \omega(\alpha) \equiv 0(\bmod p)$. It is therefore clear that

$$
v(c) \equiv 0(\bmod p) .
$$

The proof of the theorem is now complete.

2. Applications. The purpose of this section is to consider some consequences of Theorem 1.3. We first suppose that $p=2$. Then there is no restriction on the ground field $F$, since $\zeta_{p}=-1$ always belongs to $F$. Fröhlich [1] defined the discriminant field $\Omega / F$ of an extension $K / F$ as a quadratic subfield ( $\Omega=F$ possible) uniquely characterized by the relation

$$
d(K / F) \cdot J_{F}^{2}=d(\Omega / F) \cdot J_{F}^{2} .
$$


Hence $\Omega=F\left(\theta^{1 / 2}\right)$. We use the properties of $\Omega$ to prove

THEOREM 2.1. The 2-Sylow groups of the Galois group $G$ of an even degree extension $K / F$ are cyclic if and only if $d(K / F) \in J_{F}^{2} / U_{F}^{2}$.

Proof. Suppose a 2-Sylow subgroup $G_{2}$ is cyclic. Then $G_{2}$ has a normal 2-complement $N$ so that $G / N \cong G_{2}$. Let $L$ be the fixed field of $N$. Then the tower formula yields $d(L / F) J_{F^{\prime}}^{2}=d(\Omega / F) J_{F}^{2}$, so that by Fröhlich's characterization, $\Omega \subset L$.

Now $\theta \equiv 1\left(\bmod F^{2}\right)$ implies that almost all $v$ split in $L$, whence $G_{2}$ cannot be cyclic. The converse, of course, is contained in Theorem 1.3 .

REMARK. An independent proof is given in [2]. Also, a proof when $G$ is abelian appears in [6].

We now prove two further results for $p=2$.

THEOREM 2.2. If $K / F$ is normal and nonramified, and $G$ contains a noncyclic 2-Sylow group, then $\mathfrak{D}_{K}$ has an $\mathfrak{D}_{\mu}$-integral basis.

Proof. Immediate from Theorem 2.1 and Theorem 2.5 of [1].

THeOREM 2.3. If $K / F$ is a Galois extension and $d(K / F) \notin J_{F}^{2} / U_{F}^{2}$, then $G$ is solvable.

Proof. Since $\theta$ is not a square, the degree $(K: F)$ must be even since $(\Omega: F)=2$. Therefore by Theorem 2.1 the 2-Sylow groups are cyclic. Hence any such subgroup $G_{2}$ has a normal 2-complement $N$ with $G / N \cong G_{2}$. Since both $N$ and $G_{2}$ are solvable, $G$ is itself solvable.

For the remainder of the section, consider an arbitrary prime $p \geqq 2$. This now imposes a restriction on $F$. Moreover, if $p>2$ then $\theta$ is not determined, up to a $p$ th power, by $d(K / F)$, as was the case when $p=2$. Hence the notion of a discriminant field does not extend to an arbitrary prime. Also, the exceptional primes, which play no role in the $p=2$ theory, are now important. The results for $p>2$ are therefore not as strong as these obtained for $p=2$.

However, we have the following generalization of Herbrand's theorem.

THEOREM 2.4. Assuming the hypotheses of Theorem 1.3, then $\mathfrak{D}_{K / F}$ can be written as a product of ideals in the form $\mathfrak{Y}^{p} \mathfrak{D}(\theta)$, where $\theta \equiv 1(\bmod \mathfrak{B})$, and $\mathfrak{B}$ is the greatest divisor of $\left(\zeta_{p}-1\right)^{p}$, prime to $\mathrm{D}_{K / F}$; D is divisible only by ramified primes and is characterized by the relations 


$$
v(D)= \begin{cases}\dot{\phi}_{K / F}(v) & \text { if } v \text { is exceptional } \\ -v(\theta)-\frac{(L: F)}{e_{L / F}(v)} & \text { if } v \text { is ramified, nonexceptional }\end{cases}
$$

Proof. In the representation of Theorem 1.3, let the idèle $d$ be defined by $d_{v}=1$ at all infinite divisors, and $d_{v}=\pi_{v}^{\phi(v)} \theta^{\gamma(v)-1}$ at all $v \in \mathscr{C}_{F}$. Let $\mathfrak{D}$ be the ideal naturally determined by $d$; then $v(\mathscr{D})=\phi(v)+$ $v(\theta)(\psi(v)-1)$. The computations are straightforward, using the congruence relation at the end of the previous section.

Since $\phi \equiv 0$ and $\psi \equiv 1$ when $p=2$, it is evident that, for $v \in \mathscr{l}_{F}$ at least, this result is consistent with Herband's theorem.

If the exceptional divisors are known, $b$ can be determined from $d(K / F)$, for a consequence of the representation theorem is that for an exceptional divisor $v, \phi_{K / F}(v) \equiv d(K / F)(\bmod p)$. In this case, the next result gives a sufficient condition for $G_{p}$ to be cyclic.

THEOREM 2.4. Under the hypotheses of Theorem 1.3, suppose that

$$
d(K / F) \equiv a_{1}^{p} b c_{1}\left(\bmod U_{F}^{2}\right),
$$

where $b$ is as determined in Theorem 1.3. Then $G_{p}$ is cyclic if $c_{1} \notin$ $U_{F}^{2} J_{F}^{p}$. If $K / F$ is a cyclic p-extension, then a necessary condition for $u$ to split in $K$ is that $c_{1,} \in U^{2} F_{v}^{p}$.

Proof. Let $c$ be determined as in Theorem 1.3. Then $c_{1} \equiv c$ $\left(\bmod U_{F}^{2} J_{F}^{p}\right)$. If $G_{p}$ is noncyclic, then $c=1$, whence $c_{1} \in U_{F}^{2} J_{F}^{p}$. Now if $K / F$ is a cyclic $p$-extension, then $\theta \in F_{v}^{p}$ if and only if $v$ splits in $K$, whence $c_{1,} \in U_{v}^{2} F_{v}^{p}$ if $v$ splits.

The results of this section show how $d(K / F)$ can be used to obtain structural information about the Galois group of $K / F$, or in the case of cyclic $p$-extensions, the splitting of primes.

\section{REFERENCES}

1. A. Fröhlich, Discriminants of algebraic number fields, Math. Zeitschr., 74 (1960), 18-28.

2. V. Gallagher, The trace-form on Galois field extensions, pre-print.

3. E. Hecke, Vorlesungen uber die Theorie der algebraischen Zahlen, New York, 1948.

4. J. Herbrand, Une propriété du discriminant des corps algébriques, Ann. École normale (3), 49 (1932), 105-112.

5. R. Mackenzie and G. Whaples, Artin-Schreier equations in characteristic zero, Amer. J. Math., 78 (1956), 473-485.

6. D. Maurer, Invariants of the trace-form of a number field, Linear and Multilinear Algebra, 6 (1978), 33-36.

7. J. P. Serre, Corps Locaux, Paris, 1968. 
Received June 24, 1977 and in revised form March 9, 1979.

Center for Naval Analyses

2000 N. BEAUREGARD ST.

ALEXANDRIA, VA 22331 



\section{PACIFIC JOURNAL OF MATHEMATICS}

\section{EDITORS}

DONALD BABBITT (Managing Editor)

University of California

Los Angeles, CA 90024

HUgo RossI

University of Utah

Salt Lake City, UT 84112

C. C. Moore and ANDrew OGG

University of California

Berkeley, CA 94720

\section{J. DUGUNDJI}

Department of Mathematics

University of Southern California

Los Angeles, CA 90007

R. FinN and J. MILGRaM

Stanford University

Stanford, CA 94305

\section{ASSOCIATE EDITORS}
E. F. BECKENBACH
B. H. NeumanN
F. WOLF
K. YOSHIDA

\section{SUPPORTING INSTITUTIONS}

UNIVERSITY OF BRITISH COLUMBIA CALIFORNIA INSTITUTE OF TECHNOLOGY

UNIVERSITY OF CALIFORNIA

MONTANA STATE UNIVERSITY

UNIVERSITY OF NEVADA, RENO

NEW MEXICO STATE UNIVERSITY

OREGON STATE UNIVERSITY

UNIVERSITY OF OREGON
UNIVERSITY OF SOUTHERN CALIFORNIA

STANFORD UNIVERSITY

UNIVERSITY OF HAWAII

UNIVERSITY OF TOKYO

UNIVERSITY OF UTAH

WASHINGTON STATE UNIVERSITY

UNIVERSITY OF WASHINGTON

The Supporting Institutions listed above contribute to the cost of publication of this Journal, but they are not owners or publishers and have no responsibility for its content or policies.

Mathematical papers intended for publication in the Pacific Journal of Mathematics should be in typed form or offset-reproduced, (not dittoed), double spaced with large margins. Please do not use built up fractions in the text of the manuscript. However, you may use them in the displayed equations. Underline Greek letters in red, German in green, and script in blue. The first paragraph or two must be capable of being used separately as a synopsis of the entire paper. Please propose a heading for the odd numbered pages of less than 35 characters. Manuscripts, in triplicate, may be sent to any one of the editors. Please classify according to the scheme of Math. Reviews, Index to Vol. 39. Supply name and address of author to whom proofs should be sent. All other communications should be addressed to the managing editor, or Elaine Barth, University of California, Los Angeles, California, 90024.

50 reprints to each author are provided free for each article, only if page charges have been substantially paid. Additional copies may be obtained at cost in multiples of 50 .

The Pacific Journal of Mathematics is issued monthly as of January 1966. Regular subscription rate: $\$ 84.00$ a year (6 Vols., 12 issues). Special rate: $\$ 42.00$ a year to individual members of supporting institutions.

Subscriptions, orders for numbers issued in the last three calendar years, and changes of address should be sent to Pacific Journal of Mathematics, P.O. Box 969, Carmel Valley, CA 93924, U.S.A. Older back numbers obtainable from Kraus Periodicals Co., Route 100, Millwood, NY 10546.

PUBLISHED BY PACIFIC JOURNAL OF MATHEMATICS, A NON-PROFIT CORPORATION

Printed at Kokusai Bunken Insatsusha (International Academic Printing Co., Ltd.). 8-8, 3-chome, Takadanobaba, Shinjuku-ku, Tokyo 160, Japan.

Copyright (C) 1979 by Pacific Journal of Mathematics Manufactured and first issued in Japan 


\section{Pacific Journal of Mathematics}

\section{Vol. 85, No. $2 \quad$ October, 1979}

Charles A. Akemann and Steve Wright, Compact and weakly compact derivations of $C^{*}$-algebras ........................... 253

Dwight Richard Bean, Andrzej Ehrenfeucht and George Frank McNulty, Avoidable patterns in strings of symbols ................... 261

Richard Clark Brown, Notes on generalized boundary value problems in Banach spaces. I. Adjoint and extension theory.................

Kenneth Alexander Brown and John William Lawrence, Injective hulls of group rings .................................... 323

Jacob Burbea, The Schwarzian derivative and the Poincaré metric ....... 345

Stefan Andrus Burr, On the completeness of sequences of perturbed polynomial values ....................................

Peter H. Chang, On the characterizations of the breakdown points of quasilinear wave equations..............................

Joseph Nicholas Fadyn, The projectivity of $\operatorname{Ext}(T, A)$ as a module over

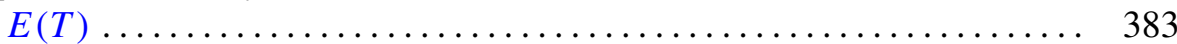

Donald Eugene Maurer, Arithmetic properties of the idèle discriminant .... 393

Stuart Rankin, Clive Reis and Gabriel Thierrin, Right subdirectly irreducible semigroups ................................. 403

David Lee Rector, Homotopy theory of rigid profinite spaces. I ........ 413 Raymond Moos Redheffer and Wolfgang V. Walter, Comparison theorems for parabolic functional inequalities................

H. M. (Hari Mohan) Srivastava, Some generalizations of Carlitz's theorem

James Alan Wood, Unbounded multipliers on commutative Banach algebras....

T. Yoshimoto, Vector-valued ergodic theorems for operators satisfying norm

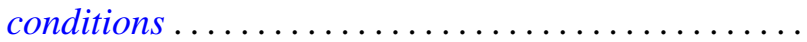

Jerry Searcy and B. Andreas Troesch, Correction to: "A cyclic inequality and a related eigenvalue problem".

Leslie Wilson, Corrections to: "Nonopenness of the set of Thom-Boardman maps" 